\title{
A single 3D chromatin compartment in amphioxus reveals stepwise evolution of vertebrate Hox bimodal regulation
}

\author{
Rafael D. Acemel ${ }^{1,5}$, Juan J. Tena ${ }^{1,5}$, Ibai Irastorza ${ }^{1,5}$, Ferdinand Marlétaz ${ }^{2,5}$, Carlos Gómez- \\ Marín $^{1}$, Elisa de la Calle-Mustienes ${ }^{1}$, Stéphanie Bertrand ${ }^{3}$, Sergio G. Diaz ${ }^{1}$, Daniel Aldea ${ }^{3}$, Jean- \\ Marc Aury ${ }^{4}$, Sophie Mangenot ${ }^{4}$, Peter W. H. Holland ${ }^{2}$, Damien P. Devos ${ }^{1,6}$, Ignacio Maeso ${ }^{1,6}$, \\ Hector Escrivá ${ }^{3,6}$, José Luis Gómez-Skarmeta ${ }^{1,6}$
}

${ }^{1}$ Centro Andaluz de Biología del Desarrollo (CABD), Consejo Superior de Investigaciones Científicas/Universidad Pablo de Olavide, Sevilla, Spain.

${ }^{2}$ Department of Zoology, University of Oxford, Oxford, United Kingdom.

${ }^{3}$ UPMC Univ Paris 06, CNRS, UMR 7232, BIOM, Observatoire Océanologique de Banyuls sur Mer, Banyuls/Mer, France.

${ }^{4}$ Commissariat à l'Energie Atomique (CEA), Institut de Génomique (IG), Genoscope, Evry, France.

${ }^{5}$ These authors contributed equally to this work

${ }^{6}$ Correspondence to: jlgomska@upo.es, hescriva@obs-banyuls.fr, nacho.maeso@gmail.com, dpdev@upo.es 
The HoxA and HoxD gene clusters of jawed vertebrates are organized into bipartite 3D chromatin structures that separate long-range regulatory inputs coming from the anterior and posterior Hox neighboring regions ${ }^{1}$. This architecture is instrumental in allowing vertebrate Hox genes to pattern disparate parts of the body, including limbs ${ }^{2}$. Almost nothing is known about how these 3D topologies originated. Here, we perform an extensive 4C-seq profiling of the Hox cluster in embryos of amphioxus, an invertebrate chordate. We find that, in contrast to vertebrates, the amphioxus Hox cluster is organized into a single chromatin interaction domain that includes long-range contacts mostly from the anterior side, bringing distant cis-regulatory elements into contact with Hox genes. We infer that the vertebrate Hox bipartite regulatory system is an evolutionary novelty built by combining ancient long-range regulatory contacts from DNA in the anterior Hox neighborhood with new regulatory inputs from the posterior side.

How the 3D organization of DNA in the nucleus impacts on regulation of gene expression is a topic of central importance in biology ${ }^{3}$. Despite recent progress in understanding chromatin organization, little is known about how such functional interactions evolve. Here, we study the evolutionary pathway leading to the bipartite $3 \mathrm{D}$ chromatin architecture regulating vertebrate Hox gene expression. In animals, chromatin is compartmentalized into Topological Associating Domains (TADs): megabase-scale chromatin regions within which DNA sequences preferentially interact with one another ${ }^{4,5}$. A paradigmatic example of how TADs organize gene regulatory information is presented by the vertebrate Hox clusters, which contain genes of pivotal importance for animal development ${ }^{6}$. Different chromosome conformation capture techniques have revealed that HoxA and HoxD genomic regions are each divided into two main adjacent TADs. These TADs compartmentalize long-range regulatory inputs coming from either side of the clusters into two major domains: enhancers distal to the 3' flank preferentially contact 'anterior' Hox genes whereas those beyond the 5 ' mostly interact with 'posterior' genes ${ }^{2,7-10}$, Fig. 1a). This bipartite regulatory topology provides gnathostomes with a versatile bimodal system allowing Hox genes to pattern multiple structures, including an ancestral role in anteroposterior axis patterning and novel roles in morphological innovations such as paired limbs ${ }^{1}$. 
To address whether TADs associated with HoxA and HoxD arose independently or have a shared ancestry dating to before the two vertebrate-specific genome duplications (2R WGDs, Supplementary Figure $1^{11}$ ), we first studied synteny conservation around Hox clusters between and within species. In mouse, HoxA and HoxD neighboring regions are strikingly different, with many HoxA long-range cis-regulatory elements (CREs) embedded in introns of neighboring genes, but HoxD long-range CREs located in gene deserts (i.e. large intergenic regions devoid of coding genes). Data from divergent vertebrates, including elephant shark, show that this is a derived situation and all vertebrate Hox cluster neighborhoods were originally very similar. What is now a HoxD gene desert in mammals contained copies of HoxA neighboring genes ${ }^{12}$, and gene-free regions surrounding the other two Hox clusters have also resulted from differential loss of neighboring genes coding exons ${ }^{13}$ (Fig. 1b, Supplementary Figure 2, Supplementary Note). Thus, differences in the genomic organization of mammalian HoxA and HoxD regulation are derived, not ancestral. This implies that CREs currently engaged in Hox long-range bipartite contacts were primarily intronic and intergenic within a conserved array of neighboring proteincoding loci before Hox cluster duplications (Fig. 1b).

We investigated the ancestry of this arrangement examining the location of vertebrate Hox-neighboring genes in invertebrate genomes. We find that few of these homologues are closely linked to Hox clusters outside chordates, and that gene order and orientations are highly variable (e.g. vertebrate anterior-linked genes are frequently found at the posterior sides and the other way round Supplementary Figure 3). This shuffling of the Hox syntenic environment suggests that in the bilaterian ancestor, long-range Hox cis-regulatory interactions were either absent or not important enough to constraint microsynteny. In contrast, in amphioxus (a nonvertebrate chordate that retains many ancestral genomic and morphological features; ${ }^{14-16}$ ), synteny on the anterior side of the Hox cluster is strikingly conserved with vertebrates; gene order and orientations are almost identical to those inferred for the vertebrate ancestor (Fig. 1b). On the posterior side, most neighboring genes are different: only two immediately adjacent genes, Evx and Lnp, are conserved in position. The conservation of anterior flanking genes between vertebrates and amphioxus suggests that long-range regulatory interactions from the 3' side had become essential for Hox regulation at the base of the chordate lineage, imposing strong constraints to genomic rearrangements in this region. In the case of the posterior side, given the lack of synteny conservation in non-chordate animals, at present, we cannot discern whether 
amphioxus or vertebrates have diverged the most from the syntenic organization of the chordate ancestor. Whatever the case, beyond Evx and Lnp, gene synteny has followed different evolutionary routes in two chordate groups, suggesting that, in stark contrast to anterior territories, the regulatory contribution of distant posterior regions was less important or even absent in their last common ancestor.

To evaluate this hypothesis experimentally, we compared Hox chromatin contacts between amphioxus and vertebrate embryos using circular chromosome conformation capture followed by high-throughput sequencing (4C-seq), a method that reveals distal chromatin contacts. Studies in mouse embryonic tissues and whole zebrafish embryos have demonstrated that 4C-seq efficiently resolves the organization of the HoxA and HoxD long-range contacts into two adjacent TADs ${ }^{2,7,8,10,17}$. We generated $4 \mathrm{C}$-seq data for fourteen gene 'viewpoints' (8 Hox genes and 6 neighboring genes) in amphioxus embryos and compared these results with previously reported ${ }^{8}$ and newly generated zebrafish data (4 Hox genes and 5 neighboring genes). In total, 73 4C-seq datasets were generated including replicates for all viewpoints and 3 amphioxus developmental stages (see Online Methods).

With these datasets, we first defined target interacting regions for each of the $4 \mathrm{C}$-seq viewpoints (genomic regions showing a statistically significant read enrichment against a randomized background) and quantified the number of reads corresponding to each of these targets (Online Methods). These analyses revealed the characteristic bipartite distribution of anterior and posterior Hox genes long-range contacts previously reported in mouse and zebrafish 2,8,10,18 (Fig. 2a, Supplementary Figure 4). Zebrafish hoxd4a and hoxd13a show little contact overlap, with the majority of their interactions mapping towards opposing sides of the cluster (83.3\% anterior and $76.6 \%$ posterior, respectively). In contrast, in amphioxus, Hox genes located at the edges of the cluster show the opposite trend: most Hox2 and Hox15 contacts converge towards the same direction, with their interacting regions located primarily within the Hox complex ( $75.2 \%$ and $74.2 \%$ respectively). In fact, regardless of their position within the cluster, anterior, central and posterior Hox genes exhibit 4C-seq profiles that overlap extensively, with no signs of a bipartite distribution (Fig. 2b, Supplementary Figure 5). Importantly, these Hox interaction profiles are developmentally stable, even though the number of active Hox genes in amphioxus changes dramatically from early gastrula to premouth embryo ${ }^{19}$ (Supplementary Figure 6). This temporal stability is in line with previous findings in mouse and Drosophila, 
where most long-range 3D chromatin interactions are organized similarly across tissues and developmental stages, with only some differences in the intensity of the contacts upon activation of different sets of distal enhancers ${ }^{7,20,21}$. However, despite this temporal uniformity, it is conceivable that in amphioxus TAD structures could be less similar across cell populations with different transcriptional activities than they are in vertebrates; thus, by using whole embryos we may be missing cell type-specific chromatin interactions.

We then correlated 4C-seq results with synteny data. Consistent with the high conservation of anterior neighboring genes, in the majority of amphioxus Hox viewpoints, a significant fraction of contacts map to the conserved anterior region (ranging from 14 to $24.8 \%$ for the promoters of Hox2, Hox5, Hox6, Hox7 and Hox9 Supplementary Table 1). Long-range interactions between Hox genes and anterior territories are even clearer when using 3' neighboring genes as viewpoints (Supplementary Figure 5, Supplementary Table 1). The amphioxus cluster contains $25.5 \%$ of Hnrnpa interactions, a similar fraction than its ortholog in zebrafish (33.4\%), and in the case of amphioxus Mtx2, the percent of contacts corresponding to the Hox complex reaches $42.7 \%$. In contrast, at the posterior side, we found striking differences between amphioxus and vertebrates. Hox genes contact posterior neighboring regions in both chordate lineages; however, the distribution of these 5' interactions is very different (Fig. 2a-b). In zebrafish, hoxd13a interactions enter into far distant 5' territories, well beyond the evx2-Inpa syntenic region, reaching vertebrate-specific posterior neighboring genes such as atp5g3a and creb2, consistent with previous reports on the location of zebrafish and mouse 5' long-range Hox enhancers ${ }^{7,22,23}$. In amphioxus, by contrast, the target interacting regions of the posterior-most Hox gene, Hox15, are circumscribed to the most proximal neighboring region, with no significant contacts crossing the Lnp promoter towards the amphioxus-specific territory. Thus, even within the only 5 ' region with synteny conservation, interaction profiles are different. In both cases, the Evx-Lnp contacts Hox genes, but while in amphioxus Evxa and Lnp show a clear interaction preference towards the Hox cluster side $(66.1 \%$ and $73 \%$, respectively), zebrafish evx2 and Inpa preferentially contact vertebrate specific genomic regions (with only $26.8 \%$ and $20.7 \%$ of the contacts towards the cluster, respectively)(Supplementary Figures 4-5, Supplementary Table 1). Taken together, these results suggest that there is an inflexion point for long-range chromatin interactions around the Evxa-Evxb-Lnp region in amphioxus, with no significant Hox-contacts with 5' amphioxus-specific genes. 
To better characterize vertebrate and amphioxus Hox topologies and identify interaction compartments, we generated virtual 3D chromatin architecture models using read counts of the 4C-seq signals as a proxy to distance from each viewpoint (Supplementary Figure 7 and Online Methods). As 4C-seq data correspond to pooled cells from whole embryos, our 3D models provide an average view of chromatin topologies, rather than the dynamic chromatin folding present in each individual cell. These integrative visualizations emphasize how strikingly different the vertebrate and amphioxus 3D chromatin architectures are (Fig. 3). In zebrafish, the HoxDa cluster sits between the two separate anterior and posterior chromatin domains; like a hinge on which the two sets of long-range regulatory inputs can swing. In contrast, the amphioxus Hox cluster appears as a large single chromatin domain that contains distant anterior neighboring genes but not posterior ones. To visualize boundaries between these chromatin domains, we developed a new approach to transform our 4C-seq-derived 3D modeling data into a heatmap of distances (analogous to those obtained by Hi-C, hereafter termed virtual Hi-C; see Online Methods and Supplementary Figures 8-10 for details on virtual Hi-C validations). As expected, zebrafish virtual $\mathrm{Hi}-\mathrm{C}$ recovered the bipartite architecture that divides vertebrate HoxD clusters into the anterior and posterior TADs (Fig. 3b). In contrast, the amphioxus cluster is contained within a single TAD that includes the conserved anterior neighboring genes, but not the amphioxus-specific posterior genes (such as Gpatch8, which has its own interacting compartment) (Fig. 3d). Importantly, no boundaries bisect the cluster or separate Hox genes from anterior neighboring territories. In the case of Lnp and the amphioxus Evx genes the situation is less clear: although these loci seem to be part of their own small interaction domain, this region is not completely isolated from its two adjacent compartments (the one containing the Hox and the one including Gpatch8). This suggests the single Hox 3D chromatin domain present in amphioxus has a weaker contact border at its posterior side than at its anterior region, and that the EvxA-EvxB-Lnp territory can be considered as an extended boundary region (Fig. 3d).

To examine the functional significance of amphioxus Hox chromatin organization, we searched for putative enhancers active in $36 \mathrm{hpf}$ amphioxus embryos (i.e. immediately preceding what can be regarded as a pharyngula stage in amphioxus, equivalent to the zebrafish phylotypic stage at $24 \mathrm{hpf}$ ) using ATAC-seq (Assay for Transposase-Accessible Chromatin using sequencing ${ }^{24}$; Fig. 4a). In agreement with the 3D chromatin topologies inferred from the virtual Hi-C results, the distribution of ATAC-seq peaks at either side of the amphioxus Hox gene 
cluster revealed very different regulatory potentials for the two Hox neighboring regions (Supplementary Figure 11). While anterior territories are rich in putative distal enhancer regions, the posterior side contains comparatively much fewer ATAC-seq peaks. In fact, apart from the peaks tightly associated to the Evx genes or directly overlapping with transcriptional start sites and repetitive elements, we only found a single candidate enhancer region, within the intergenic region between Evxb and Lnp. We then tested four putative enhancer elements from within the TAD on the anterior side of the amphioxus cluster located at different distances from the closest Hox gene (elements 1655, 1739, 1784 and 1801, located 150kb, 66kb, 20kb and 3kb downstream of Hox1) and the aforementioned element identified at the posterior side (element 2473, $165 \mathrm{~kb}$ upstream of Hox15), by generating GFP-reporter zebrafish stable transgenes. All anterior enhancers promoted expression in the antero-posterior axis, consistent with expression patterns of amphioxus Hox genes but not with those of neighboring loci (Fig. 4b, Supplementary Figure 12 and ${ }^{19}$ ), suggesting that they are amphioxus Hox CREs. In contrast, the 2473 posterior element activates GFP expression in isolated neurons in the spinal cord, in a pattern reminiscent

of the amphioxus Evxa gene (Fig. $4 \mathrm{~b},{ }^{25}$ ) rather than a Hox gene. These experiments suggest that the $3 \mathrm{D}$ organization identified using $4 \mathrm{C}$-seq and modeling brings long-range regulatory elements into proximity with amphioxus Hox genes mostly on the anterior side of the cluster (Fig. 4).

In summary, our results support a stepwise evolution of the bimodal regulatory machineries of HoxA and D clusters of jawed vertebrates (Fig. 4c). The relatively simple Hox cluster 3D topology of early bilaterian animals, where external long-range regulation was probably absent, changed profoundly in early chordate evolution, with newly incorporated distal regulatory inputs from anterior neighboring loci becoming a fundamental part of the Hox regulatory architecture. This unipolar topology was further developed in the vertebrate lineage. The acquisition of distal CREs interactions at the posterior side permitted the switch between two separate sets of long-range regulatory inputs, allowing an unprecedented plasticity in the developmental usage of the Hox patterning system in vertebrates.

\section{Accession codes}

Data sets presented in this study are available with accession GEO number: GSE68737.

\section{Acknowledgments}


We specially thank Dr. Juan Pascual-Anaya for helping with some figures and helpful discussions. We would also like to thank Dr. Fernando Casares, Dr. Isabel Almudí and Dr. Juan Ramón Martínez-Morales for fruitful discussions. Work was funded by grants from Ministerio de Economía y Competitividad (BFU2013-41322-P to JLG-S, Juan de la Cierva postdoctoral contract to IM); the Andalusian Government (BIO-396 to JLG-S; C2A (EE: 2013/2506) to DPD and II); European Research Council (ERC Grant N ${ }^{\circ}$ 268513) to PWH and FM; EMBO short fellowship to IM; Universidad Pablo de Olavide to JJT and Conicyt "Becas Chile" to DA.

\section{Author Contributions}

R.D.A. carried out the 4C-seq experiments with the help of C.G.-M. and S.G.D. J.J.T. performed the bioinformatic analysis of all the 4C-seq and ATAC-seq datasets. I.I. developed and applied the 3D modeling and virtual Hi-C procedures. F.M. generated the assembly and annotation of the Hox locus in the European amphioxus. J.M.A. and S.M. ensured sequencing project management at Génoscope. E.d.1.C.-M., R.D.A., J.J.T. and I.M. carried out the zebrafish reporter assays. S.B. and D.A. collected and processed the amphioxus embryonic material and performed in situ hybridizations. I.M. completed the amphioxus ATAC-seq experiments and the evolution of synteny analyses. J.L.G.-S., H.E., I.M. and D.D. conceived, designed and coordinated the project. J.L.G.-S. and I.M. wrote the manuscript with the help of P.W.H.H. All the authors revised and contributed to the final version of the text.

All experimental procedures using vertebrates were ethically approved by the Andalusian Government.

\section{References}

1. Lonfat, N. \& Duboule, D. Structure, function and evolution of topologically associating domains (TADs) at HOX loci. FEBS Lett (2015).

2. Andrey, G. et al. A switch between topological domains underlies HoxD genes collinearity in mouse limbs. Science 340, 1234167 (2013).

3. de Laat, W. \& Duboule, D. Topology of mammalian developmental enhancers and their regulatory landscapes. Nature 502, 499-506 (2013).

4. Gomez-Diaz, E. \& Corces, V.G. Architectural proteins: regulators of 3D genome organization in cell fate. Trends Cell Biol 24, 703-11 (2014).

5. Ciabrelli, F. \& Cavalli, G. Chromatin-driven behavior of topologically associating domains. J Mol Biol 427, 608-25 (2015).

6. Mallo, M. \& Alonso, C.R. The regulation of Hox gene expression during animal development. Development 140, 3951-63 (2013). 
7. Montavon, T. et al. A regulatory archipelago controls Hox genes transcription in digits. Cell 147, 1132-45 (2011).

8. Woltering, J.M., Noordermeer, D., Leleu, M. \& Duboule, D. Conservation and divergence of regulatory strategies at Hox Loci and the origin of tetrapod digits. PLoS Biol 12, e1001773 (2014).

9. Berlivet, S. et al. Clustering of tissue-specific sub-TADs accompanies the regulation of HoxA genes in developing limbs. PLoS Genet 9, e1004018 (2013).

10. Lonfat, N., Montavon, T., Darbellay, F., Gitto, S. \& Duboule, D. Convergent evolution of complex regulatory landscapes and pleiotropy at Hox loci. Science 346, 1004-6 (2014).

11. Dehal, P. \& Boore, J.L. Two rounds of whole genome duplication in the ancestral vertebrate. PLoS Biol 3 , e314 (2005).

12. Lehoczky, J.A., Williams, M.E. \& Innis, J.W. Conserved expression domains for genes upstream and within the HoxA and HoxD clusters suggests a long-range enhancer existed before cluster duplication. Evol Dev 6, 423-30 (2004).

13. Maeso, I. et al. An ancient genomic regulatory block conserved across bilaterians and its dismantling in tetrapods by retrogene replacement. Genome Res 22, 642-55 (2012).

14. Paps, J., Holland, P.W. \& Shimeld, S.M. A genome-wide view of transcription factor gene diversity in chordate evolution: less gene loss in amphioxus? Brief Funct Genomics 11, 177-86 (2012).

15. Bertrand, S. \& Escriva, H. Evolutionary crossroads in developmental biology: amphioxus. Development 138, 4819-30 (2011).

16. Holland, L.Z. \& Onai, T. Early development of cephalochordates (amphioxus). Wiley Interdiscip Rev Dev Biol 1, 167-83 (2012).

17. Noordermeer, D. et al. Temporal dynamics and developmental memory of 3D chromatin architecture at Hox gene loci. Elife 3, e02557 (2014).

18. Noordermeer, D. et al. The dynamic architecture of Hox gene clusters. Science 334, 222-5 (2011).

19. Pascual-Anaya, J. et al. Broken colinearity of the amphioxus Hox cluster. Evodevo 3, 28 (2012).

20. Dixon, J.R. et al. Topological domains in mammalian genomes identified by analysis of chromatin interactions. Nature 485, 376-80 (2012).

21. Ghavi-Helm, Y. et al. Enhancer loops appear stable during development and are associated with paused polymerase. Nature 512, 96-100 (2014).

22. Gonzalez, F., Duboule, D. \& Spitz, F. Transgenic analysis of Hoxd gene regulation during digit development. Dev Biol 306, 847-59 (2007).

23. Gehrke, A.R. et al. Deep conservation of wrist and digit enhancers in fish. Proc Natl Acad Sci U S A 112, 803-8 (2015).

24. Buenrostro, J.D., Giresi, P.G., Zaba, L.C., Chang, H.Y. \& Greenleaf, W.J. Transposition of native chromatin for fast and sensitive epigenomic profiling of open chromatin, DNA-binding proteins and nucleosome position. Nat Methods 10, 1213-8 (2013).

25. Ferrier, D.E., Minguillon, C., Cebrian, C. \& Garcia-Fernandez, J. Amphioxus Evx genes: implications for the evolution of the Midbrain-Hindbrain Boundary and the chordate tailbud. Dev Biol 237, 270-81 (2001). 


\section{Figures}

Fig. 1. Genomic organization of vertebrate and amphioxus Hox clusters. (a) Distribution of TADs (obtained from human cells Hi-C datasets ${ }^{20}$ ) and schematics of the chromatin architecture of HoxA and HoxD clusters, showing their similar 3D topology. Colored bars represent Hox (white), anterior (blue) and posterior (red) neighboring genes. (b) Microsynteny arrangements around the Hox clusters of gnathostomes, the pre-WGD vertebrate ancestor and amphioxus. Genes are represented by arrows according to the transcriptional orientation (white, Hox clusters; blue, anterior; red, posterior; grey, genes with non-conserved linkages), those outlined by dashed lines correspond to vertebrate paralogs that have been lost in at least one species. Question marks indicate genes whose status in the vertebrate ancestor could not be inferred. Slashes correspond to non-conserved amphioxus loci shown in Supplementary Figure 3.

Fig. 2. Comparative of the 4C-seq interaction profiles of zebrafish and amphioxus Hox clusters. Normalized 4C-seq profiles of several Hox genes promoters in zebrafish HoxDa locus (a) and amphioxus Hox locus (b). Spider plots show the statistically significant contacts to the left (blue arcs) and to the right (red arcs) of each viewpoint. Percentages of reads aligned to statistically significant targets at each side of viewpoints are indicated in blue (left contacts) or red (right contacts). Units in the y-axes correspond to normalized interacting counts. Green bars indicate the positions of the viewpoints.

Fig. 3. 3D chromatin architecture of amphioxus and zebrafish Hox clusters. 3D models of zebrafish HoxDa (a) and amphioxus Hox (c) regions. 4C-seq viewpoints are highlighted (blue, anterior genes; yellow, Hox genes; red, posterior genes). (b, d) Zebrafish and amphioxus virtual Hi-C consensus of all 3D model solutions. 4C-seq viewpoints are represented by circles with the same color scheme of the previous panels. Arrows point out the TAD border bisecting the zebrafish Hox cluster (b) and the absence of this border in the case of amphioxus (d).

Fig. 4. Regulatory compartments in the amphioxus Hox region and evolution of Hox 3D architecture. (a) Amphioxus virtual Hi-C heatmap and ATAC-seq profile at $36 \mathrm{hpf}$ in the Hox region. Amphioxus ATAC-seq peaks tested in zebrafish are colored and highlighted by asterisks 
(blue for those in the anterior region, red for the one at the posterior side of the cluster). (b) Lateral views of 24-hpf and 48-hpf (inset in 2473) embryos of zebrafish transgenic lines showing GFP expression driven by the amphioxus ATAC-seq peaks (1655, 1739, 1784, 1801 and 2473) highlighted in (a). Midbrain expression corresponds to the enhancer positive control included in the reporter constructs. Whole mount in situ hybridizations of Hox1 and Evxa in 36-hpf amphioxus embryos are shown for comparisons. Anterior is to the left. (ey) eye; (hb) hindbrain; (mb) midbrain; (nc) neural crest cells; (ne) neurons; (no) notochord; (ot) otic vesicle, (op) olfactory placode, (sc) spinal chord. (c) 3D-architectures schematics showing an evolutionary scenario for the origin of the bimodal regulatory system of jawed vertebrates. The Hox-only chromatin domain of early bilaterians is first expanded by the anterior side in the chordate ancestor and by the posterior side at the origin of vertebrates, allowing the bipartition of the regulatory topologies of HoxA and HoxD clusters.

\section{Online Methods}

\section{Genome sequencing and assembly}

DNA was prepared from a single European amphioxus (Branchiostoma lanceolatum) mature male and sequenced using Illumina technology at Génoscope (Centre National de Séquençage, Evry, France). Briefly, two paired-end (180bp and 700bp) and 6 mate-pair libraries $(3,5,8 \mathrm{~kb})$ were generated and sequenced at $>200 \mathrm{x}$ total coverage.

Reads were quality-trimmed using sickle (v1.290, https://github.com/najoshi/sickle), error corrected using Musket (v1.0.6) ${ }^{26}$ and overlapping libraries merged using Flash ${ }^{27}$. Assembly was carried out using SOAPdenovo (v2.04) ${ }^{28}$ using a k-mer of 71 for contiging and of 35 for mapping and scaffolding. Gaps were subsequently filled using GapCloser (v1.2) ${ }^{28}$ with an overlap parameters set to 31 . The resulting assembly (N50: 649,215, size: $948.5 \mathrm{Mb}$ ) contains allelic copies for most scaffolds (expected genome size $\sim 500 \mathrm{Mb}$ ) that we reconciled using the Haplomerger ${ }^{29}$ pipeline relying on best-reciprocal lastz alignment after masking repeats using a custom library built with Repeatmodeler (http://www.repeatmasker.org). The Hox locus was extracted from the final assembly (N50: $1132648 \mathrm{bp}$, size: 526.8Mb) and submitted together with the 4C-seq and ATAC-seq data (GSE68737). 
Gene models were built using Evidence Modeler (EVM) ${ }^{30}$ based on (i) de novo gene prediction obtained using Augustus ${ }^{31}$ with a custom training based on CEGMA ${ }^{32}$ report, (ii) split-aware alignment of human proteins using Exonerate ${ }^{33}$ and transcriptome alignment. Models for known genes within the Hox region that were not present in these annotations were added manually. More details regarding genome $B$. lanceolatum assembly and annotation will be provided in a separate upcoming publication.

\section{Synteny analyses and genome browsing}

Hox neighboring genes were searched across the different studied species using tblastn and blastp. We compared the relative orientations and positions of these genes by browsing the genomes of the studied species through the NCBI (http://www.ncbi.nlm.nih.gov/blast/Blast.cgi), UCSC (http://genome.ucsc.edu/), and Ensembl Metazoa (http://metazoa.ensembl.org/info/website/species.html) webpages, using the following genome versions: Elephant shark (Callorhinchus milii) 6.1.3, Lottia gigantea v1.0, Mus musculus Build 38, Saccoglossus kowalevskii Build1.1, Strigamia maritima Smar1.0, Trichoplax adhaerens v1.0. In the case of the starfish Acanthaster planci, no gene annotation or genome browser were available for the published A. planci Hox genome scaffold (accession number DF933567.1) ${ }^{34}$. Therefore, we used tblastn to search for conserved neighboring genes and Genscan to predict genes de novo.

Mouse Jazf2 pseudogenized exons were detected with VISTA ${ }^{35}$ using elephant shark as a reference sequence, LAGAN as the alignment program and the following parameters: $100 \mathrm{bp}$ window and $65 \%$ of identity in $70 \mathrm{bp}$.

\section{Amphioxus procurement and culture}

B. lanceolatum ripe adults were collected at the Racou beach in Argelès-sur-Mer

(France). Gametes were collected by heat stimulation as previously described ${ }^{36,37}$. Fertilization was undertaken in Petri dishes filled with filtered sea water and embryos were cultured at $19^{\circ} \mathrm{C}$.

\section{Whole mount in situ hybridization}

Partial cDNAs from Gpatch8, Nfe2, Lnp, Slc20, Mtx2, Hnrnpa, and Cbx of B. lanceolatum were amplified by RT-PCR and cloned in pGEM-T Easy vector. DIG-labeled RNA 
probes were synthesized by in vitro translation after plasmid linearization using the appropriate enzymes. Fixation and whole-mount in situ hybridization (WISH) were performed as described

in ${ }^{38}$. No expression could be detected using WISH for Gpatch8, Lnp, Slc20 and Mtx2; the expression patterns for the rest of the genes are included in Supplementary Figure 12.

\section{C-seq}

$4 \mathrm{C}$-seq assays were performed as previously reported ${ }^{18,39-41}$. For each zebrafish biological replicate, 500 embryos at $24 \mathrm{hpf}$ of the Tübingen strain were dechorionated using pronase and deyolked in $1 \mathrm{ml}$ of Ginzburg Fish Ringers (55 mM NaCl, $1.8 \mathrm{mM} \mathrm{KCl}, 1.25 \mathrm{mM} \mathrm{NaHCO}$ ). Then, they were fixed in PBS1X-formaldehyde 2\% for 15 minutes at room temperature. For amphioxus biological replicates, embryos ( $\sim 8000$ in the case of $8 \mathrm{hpf}$ and $\sim 4000$ for the $15 \mathrm{hpf}$ and $36 \mathrm{hpf}$ stages) were concentrated by centrifugation at low speed in $2 \mathrm{~mL}$ microtubes. They were fixed 15 minutes at room temperature in $1,5 \mathrm{~mL}$ of MOPS buffer $(0,1 \mathrm{M}$ MOPS $\mathrm{pH} 7,5$, $2 \mathrm{mM} \mathrm{MgSO} 2,1 \mathrm{mM}$ EGTA, $0,5 \mathrm{M} \mathrm{NaCl}$ ) containing $1,85 \%$ formaldehyde. $155 \mu \mathrm{L}$ of $10 \%$ Glycine were added to both species samples to stop the fixation, followed by 5 washes with PBS (NaPBS in the case of amphioxus) at $4^{\circ} \mathrm{C}$. Pellets were frozen in liquid nitrogen and kept at $80^{\circ} \mathrm{C}$. Isolated cells were lysed (lysis buffer: $10 \mathrm{mM}$ Tris- $\mathrm{HCl} \mathrm{pH} \mathrm{8,} 10 \mathrm{mM} \mathrm{NaCl}, 0.3 \%$ IGEPAL CA-630 (Sigma-Aldrich, I8896), 1X protease inhibitor cocktail (cOmplete, Roche, 11697498001)) and the DNA digested with DpnII (New England Biolabs, R0543M) and Csp6I (Fermentas, Thermo Scientific, FD0214) as primary and secondary enzymes respectively. T4 DNA ligase (Promega, M1804) was used for both ligation steps. Specific primers were designed around the putative transcriptional start sites of the genes with Primer3 v. 0.4.0 ${ }^{42}$. Illumina adaptors were included in the primers sequence and 8 PCRs were performed with Expand Long Template PCR System (Roche, 11759060001) and pulled together. Two libraries coming from different biological replicates were generated for each $4 \mathrm{C}$-seq experiment (i.e. for each viewpoint and for each developmental stage). These libraries were purified with a High Pure PCR Product Purification Kit (Roche, 11732668001), their concentrations measured using the Quanti-iTTM PicoGreen dsDNA Assay Kit (Invitrogen, P11496) and sent for deep sequencing. 4C-seq data were analyzed as previously described ${ }^{17}$. Briefly, raw sequencing data were demultiplexed and aligned using zebrafish July 2010 assembly (danRer7) and the B. lanceolatum reference genomes. Reads located in fragments flanked by two restriction sites of the same enzyme, in 
fragments smaller than $40 \mathrm{bp}$, or within a window of $10 \mathrm{~kb}$ around the viewpoint (indicated by dashed lines in the different figures) were filtered out. Mapped reads were then converted to reads-per-first-enzyme-fragment-end units, and smoothed using a 30 fragments mean running window algorithm. 4C-seq data were normalized by total weight of reads within the window displayed in figures.

To calculate statistically significant contacting regions for each viewpoint, an average background level was estimated as previously described ${ }^{43}$. Briefly, fragments distribution in a window of $2 \mathrm{Mb}$ around each viewpoint was randomized, excluding an internal window of 100 $\mathrm{Kb}$ around the viewpoint to avoid biases due to close contacts. Then, this randomized fragment distribution was smoothened as described above. This randomized profile was then used to calculate the p-value for each potential target in the observed 4C-seq distribution by means of Poisson probability function. Regions with p-values below 1E-5 were considered as statistically significant interacting targets.

To calculate the distribution of contacts at each side of the viewpoints we took into account only those reads overlapping the interacting targets, discarding also those mapped within the $100 \mathrm{~kb}$ viewpoint window as previously reported ${ }^{8}$. The same approach was used to quantify the distribution of contacts in the three windows defined as follows: cluster (from the 5' UTR of the 5' most Hox genes (zebrafish hoxd13a and amphioxus Hox15) to the 3' UTR of the 3' most Hox genes (zebrafish hoxd3a and amphioxus Hox1)); anterior (downstream of zebrafish hoxd3a and amphioxus Hox1); and posterior (upstream of zebrafish hoxd13a and amphioxus Hox15).

\section{D Computational Modeling and virtual Hi-C}

\section{C Data normalization}

To equal the amount of reads in all experiments, we normalized the reads of the $4 \mathrm{C}$-seq datasets. We then extracted the data relevant for the modeling by calculating the Z-score (see the sections below on Z-score thresholds optimization) of those reads as in ${ }^{44}$.

\section{Structure determination}

The overall approach of the determination of the genomes structures was adapted from a

previous work ${ }^{44}$ with some variations, using the Integrative Modeling Platform (IMP) ${ }^{45}$. The procedure was divided in three stages: 
1) Representation of the genome locus and translation of the data into spatial restraints. We represented the chromosomal fragment as a flexible string of beads where each bead corresponded to a number of consecutive fragments between 10 and 45, depending on the total size of the locus (Supplementary Figure 7c). The size of the beads representing those 20 fragments was proportional to the sum of the sizes of these fragments.

In order to impose connection between the beads, harmonic upper bound distance restraints were used between consecutive beads. This distance was the sum of the radii of both beads. Excluded volume restraints were imposed over all the beads so these would not overlap each other. The reach window of a viewpoint was defined as the area between the furthest upstream and downstream fragments with a Z-score above the upper Z-score (uZ) (Supplementary Figure 13). Harmonic distance restraints were applied between beads corresponding to the viewpoints and the rest of the beads, as long as these beads' Z-scores were above the $\mathrm{uZ}$ or below the lower Z-score (1Z). We used the absolute Z-score of the reads to give more weight to the most meaningful reads. Beads outside the reach window were restrained with harmonic lower bound distances, with a weight equal to the absolute Z-score. With the harmonic lower bound restraint we only impose the beads not to be closer than their computed distance (Supplementary Figure 7).

2) Optimization and sampling of the space of solutions. We combined a Monte Carlo exploration with a local optimization of conjugate gradients and simulated annealing. We started with an individual optimization of 5 steps of conjugate gradients from a entirely random configuration of beads followed by simulated annealing until the score difference between rounds was below 0.00001 or reached 0 (Supplementary Figure $7 \mathrm{~d}$ ). To sample the space of solutions exhaustively we computed 50.000 independent optimizations for each genome (Supplementary Figure 7e).

3) Analysis and assessment of the ensemble of models. We gathered the 200 models with the best score. Those solutions were then clustered according to their similarity measured by their Root Mean Square Deviation (RMSD). We used the Multiexperiment Viewer, MeV ${ }^{46}$ with Hierarchical Clustering and K-Means clustering. All models grouped in two clusters that were the mirror image of each other (Supplementary Figure 14). The most representative models (i.e. the closest ones to the mean of all solutions within the most populated cluster) are displayed in 
Fig. 3. Results were indistinguishable when we used the solutions of the other mirror image cluster.

Reconstruction of virtual Hi-C data

We used the models from the most populated cluster to generate the heat map plots that were equivalent to Hi-C data. First we superimposed all the models (Supplementary Figure 7f). To generate virtual Hi-C heatmap plots, we measured the distances between all beads in each model and calculated the mean of these distances (Supplementary Figure $7 \mathrm{~g}$ ).

Empirical calculation of the Maximum distance, the $\mathrm{IZ}$ and the $\mathrm{uZ}$.

The calculation of these parameters was done as described previously ${ }^{44}$ with little variations: The $\mathrm{uZ}$ varied between 0.2 and 1.4 in bins of 0.2 . The $1 \mathrm{Z}$ varied in bins of 0.2 between -1.4 and -0.2. The maximum distance varied from 3000 to 7000 in bins of 1000 . Due to the heavy computational load, we did not consider thinner bins or higher or lower values.

For each set of parameters, we generated 500 models and calculated the mean distances between the viewpoints and the rest of the fragments and compared them to the distances that represented each set of 20 fragments of the normalized 4C data (Supplementary Figure 15 b, d, f).

The set of parameters that best fitted the $4 \mathrm{C}$ data were 0.2 for the $\mathrm{uZ}$ and -0.2 for the $1 \mathrm{Z}$ in amphioxus, zebrafish and mouse. The best max distances were different for each species. To allow comparison, we needed to settle the same max distance for all three. Taking this into account, and for the sake of ease of visualization, we settled on the max distance of 7000 , whose score was also amongst the best (Supplementary Figure 15 a, c, e).

Validation of the virtual Hi-C approach

To validate the virtual Hi-C method we followed two strategies:

1) Jackknife resampling. We tested the reproducibility and robustness virtual Hi-C results taking advantage of the extensive number of viewpoints available in our amphioxus and zebrafish Hox 4C-seq data. We performed additional modeling experiments by resampling our original datasets using different subsets of $4 \mathrm{C}$ data both in zebrafish and in amphioxus (Supplementary Table 2). We generated 500 models with the same parameters that we used for our initial modeling and reconstructed virtual Hi-C data for each subset. Subsequently, we calculated Spearman's coefficients between the different subsets. This demonstrated that virtual 
$\mathrm{Hi}-\mathrm{C}$ results are very reproducible and robust to perturbations, with high correlations even when $60 \%$ of the viewpoints are eliminated (Supplementary Figure 10, Supplementary Table 2).

2) Modeling of other loci and shifted calculation of correlations. To validate the models and the virtual Hi-C derived from them, we generated models for diverse mouse genomic regions using previously published 4C-seq data ( from the HoxD locus and two additional loci: Wnt6Ihh-Epha4-Pax3 and Med13l-Tbx3-Tbx5-Rbm19 ${ }^{17,47,48}$ ). Using these models, we generated the virtual Hi-Cs and compared them with previously published experimental Hi-C data 20 (Supplementary Figures 8-9). These comparisons were performed shifting the window used for the modeling $25 \%$ of its size in each direction, in steps of $20 \mathrm{~Kb}$ (see Supplementary Figure 8 ). For each comparison, Spearman's and Pearson's correlations were calculated. Due to the dominance of read counts corresponding to short distances, we calculated these correlations using bins separated by at least 240kb (HoxD and Med13l-Tbx3-Tbx5-Rbm19) or 480kb (Wnt6Ihh-Epha4-Pax3), to account for the different size of these three loci $(\sim 2.12, \sim 2.48$ and $\sim 4.88 \mathrm{Mb}$, respectively). In all cases, our 4C-seq-derived virtual $\mathrm{Hi}-\mathrm{C}$ contact matrices accurately recapitulate the TAD organization and borders present in the experimental Hi-C maps, with Spearman's and Pearson's coefficients within the same range (from 0.63 to 0.88 ) of those

typically obtained between different Hi-C experimental conditions (from 0.4 to $0.99^{20,49-51}$ ) (Supplementary Figure 9, Supplementary Table 3).

\begin{abstract}
ATAC-seq
ATAC-seq experiments in amphioxus embryos were performed as previously described 23,24. Approximately 80000 cells (corresponding to thirteen 36 hpf embryos) were directly lysed in cold lysis buffer (10 $\mu \mathrm{M}$ Tris $\mathrm{pH} 7.4,10 \mu \mathrm{M} \mathrm{NaCl}, 3 \mu \mathrm{M} \mathrm{MgCl2}, 0.1 \%$ IGEPAL) after removing the seawater by centrifuging briefly. Then, the sample was incubated for 30 min at $37^{\circ} \mathrm{C}$ with the TDE1 enzyme and purified with Qiagen Minelute kit. A PCR was performed with 13 cycles using Ad1F and Ad2.3R primers and KAPA HiFi hotstart enzyme (Kapa Biosystems). The resulting library was multiplexed and sequenced in a HiSeq 2000 lane. Reads were aligned using the mentioned B. lanceolatum assembly. Duplicated pairs or those ones separated by more than $2 \mathrm{~Kb}$ were removed. The enzyme cleavage site was determined as the position -4 (minus strand) or +5 (plus strand) from each read start, and this position was extended 5 bp in both directions for signal visualization. For the zebrafish reporter assays of anterior elements, we
\end{abstract}


selected 4 regions including ATAC-seq peaks with no overlap with coding exons, transcriptional start sites and repetitive elements. We applied the same criteria to the posterior region, also excluding ATAC-seq peaks tightly associated with amphioxus Evx genes (i.e. those located in the Evx introns and within $5 \mathrm{~kb}$ of the Evx transcribed regions). This rendered a single candidate element between Evxb and Lnp (see Supplementary Figure 11).

\section{Transgenesis in zebrafish}

Transgenesis assays were performed as previously reported ${ }^{52}$. Putative enhancers were amplified by PCR from amphioxus genomic DNA using the primers listed in Supplementary Table 4. The PCR fragments were subcloned in PCR8/GW/TOPO vector and, using Gateway technology (Life Technologies), were shuttled into an enhancer detection vector composed of a gata2 minimal promoter, an enhanced GFP reporter gene, and a strong midbrain enhancer (z48) that works as an internal control for transgenesis in zebrafish ${ }^{23}$. Zebrafish transgenic embryos were generated using the Tol 2 transposon/transposase method ${ }^{53}$, with minor modifications. Onecell embryos were injected with a $2 \mathrm{nl}$ volume containing $25 \mathrm{ng} / \mu \mathrm{l}$ of transposase mRNA, 20 $\mathrm{ng} / \mu \mathrm{l}$ of purified constructs and $0.05 \%$ of phenol red. In order to ensure the reproducibility of the expression patterns observed in the reporter assays, three or more stable transgenic lines derived from different founders were generated for each construct.

\section{Methods-only References}

26. Liu, Y., Schroder, J. \& Schmidt, B. Musket: a multistage k-mer spectrum-based error corrector for Illumina sequence data. Bioinformatics 29, 308-15 (2013).

27. Magoc, T. \& Salzberg, S.L. FLASH: fast length adjustment of short reads to improve genome assemblies. Bioinformatics 27, 2957-63 (2011).

28. Luo, R. et al. SOAPdenovo2: an empirically improved memory-efficient short-read de novo assembler. Gigascience 1, 18 (2012).

29. Huang, S. et al. HaploMerger: reconstructing allelic relationships for polymorphic diploid genome assemblies. Genome Res 22, 1581-8 (2012).

30. Haas, B.J. et al. Automated eukaryotic gene structure annotation using EVidenceModeler and the Program to Assemble Spliced Alignments. Genome Biol 9, R7 (2008).

31. Stanke, M. et al. AUGUSTUS: ab initio prediction of alternative transcripts. Nucleic Acids Res 34, W435-9 (2006).

32. Parra, G., Bradnam, K. \& Korf, I. CEGMA: a pipeline to accurately annotate core genes in eukaryotic genomes. Bioinformatics 23, 1061-7 (2007).

33. Slater, G.S. \& Birney, E. Automated generation of heuristics for biological sequence comparison. BMC Bioinformatics 6, 31 (2005).

34. Baughman, K.W. et al. Genomic organization of Hox and ParaHox clusters in the echinoderm, Acanthaster planci. Genesis 52, 952-8 (2014). 
35. Frazer, K.A., Pachter, L., Poliakov, A., Rubin, E.M. \& Dubchak, I. VISTA: computational tools for comparative genomics. Nucleic Acids Res 32, W273-9 (2004).

36. Fuentes, M. et al. Insights into spawning behavior and development of the European amphioxus (Branchiostoma lanceolatum). J Exp Zool B Mol Dev Evol 308, 484-93 (2007).

37. Fuentes, M. et al. Preliminary observations on the spawning conditions of the European amphioxus (Branchiostoma lanceolatum) in captivity. J Exp Zool B Mol Dev Evol 302, 384-91 (2004).

38. Somorjai, I., Bertrand, S., Camasses, A., Haguenauer, A. \& Escriva, H. Evidence for stasis and not genetic piracy in developmental expression patterns of Branchiostoma lanceolatum and Branchiostoma floridae, two amphioxus species that have evolved independently over the course of 200 Myr. Dev Genes Evol 218, 703-13 (2008).

39. Dekker, J., Rippe, K., Dekker, M. \& Kleckner, N. Capturing chromosome conformation. Science 295, 1306-11 (2002).

40. Hagege, H. et al. Quantitative analysis of chromosome conformation capture assays (3C-qPCR). Nat Protoc 2, 1722-33 (2007).

41. Splinter, E., de Wit, E., van de Werken, H.J., Klous, P. \& de Laat, W. Determining long-range chromatin interactions for selected genomic sites using 4C-seq technology: From fixation to computation. Methods (2012).

42. Rozen, S. \& Skaletsky, H. Primer3 on the WWW for general users and for biologist programmers. Methods Mol Biol 132, 365-86 (2000).

43. Smemo, S. et al. Obesity-associated variants within FTO form long-range functional connections with IRX3. Nature 507, 371-5 (2014).

44. Bau, D. et al. The three-dimensional folding of the alpha-globin gene domain reveals formation of chromatin globules. Nat Struct Mol Biol 18, 107-14 (2011).

45. Russel, D. et al. Putting the pieces together: integrative modeling platform software for structure determination of macromolecular assemblies. PLoS Biol 10, e1001244 (2012).

46. Saeed, A.I. et al. TM4: a free, open-source system for microarray data management and analysis. Biotechniques 34, 374-8 (2003).

47. Lupianez, D.G. et al. Disruptions of topological chromatin domains cause pathogenic rewiring of geneenhancer interactions. Cell 161, 1012-25 (2015).

48. van Weerd, J.H. et al. A large permissive regulatory domain exclusively controls Tbx3 expression in the cardiac conduction system. Circ Res 115, 432-41 (2014).

49. Vietri Rudan, M. et al. Comparative Hi-C reveals that CTCF underlies evolution of chromosomal domain architecture. Cell Rep 10, 1297-309 (2015).

50. Hou, C., Li, L., Qin, Z.S. \& Corces, V.G. Gene density, transcription, and insulators contribute to the partition of the Drosophila genome into physical domains. Mol Cell 48, 471-84 (2012).

51. Zhang, Y. et al. Spatial organization of the mouse genome and its role in recurrent chromosomal translocations. Cell 148, 908-21 (2012).

52. Bessa, J. et al. Zebrafish enhancer detection (ZED) vector: A new tool to facilitate transgenesis and the functional analysis of cis-regulatory regions in zebrafish. Developmental Dynamics 238, 2409-2417 (2009).

53. Kawakami, K. Transgenesis and gene trap methods in zebrafish by using the Tol2 transposable element. Methods Cell Biol 77, 201-22 (2004). 
a HoxA

b
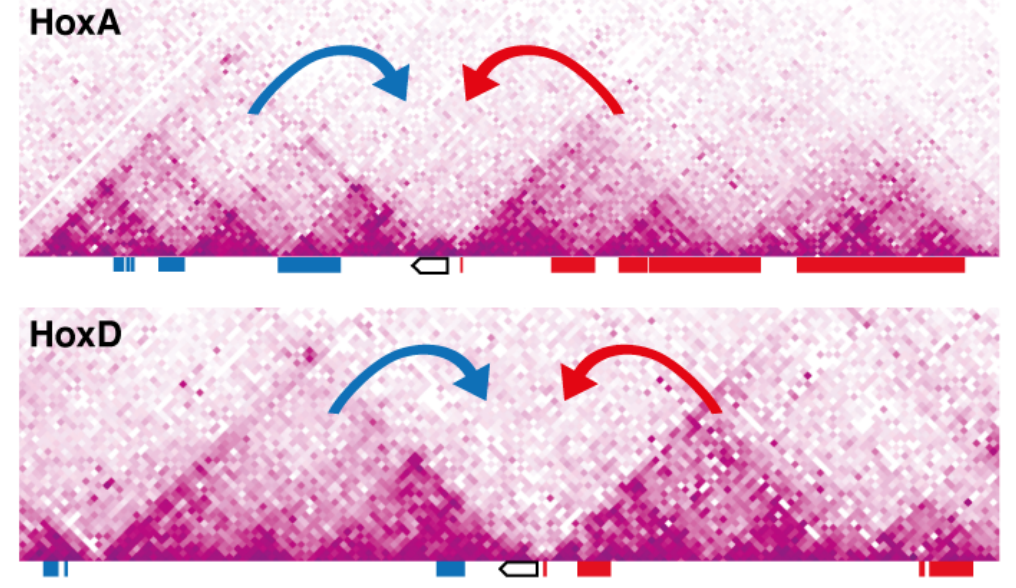
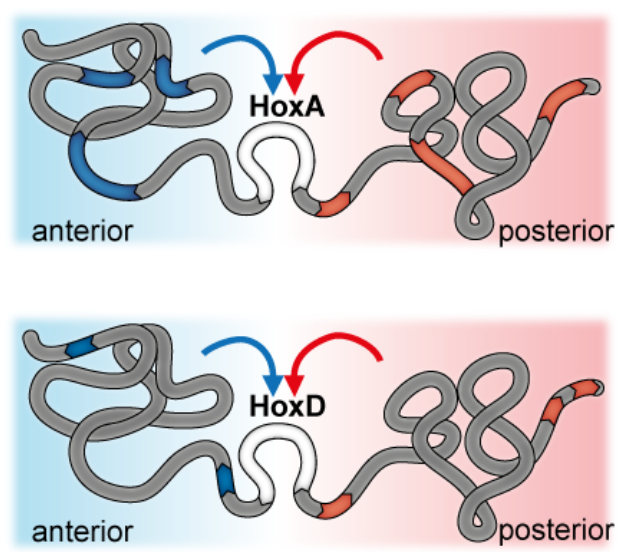

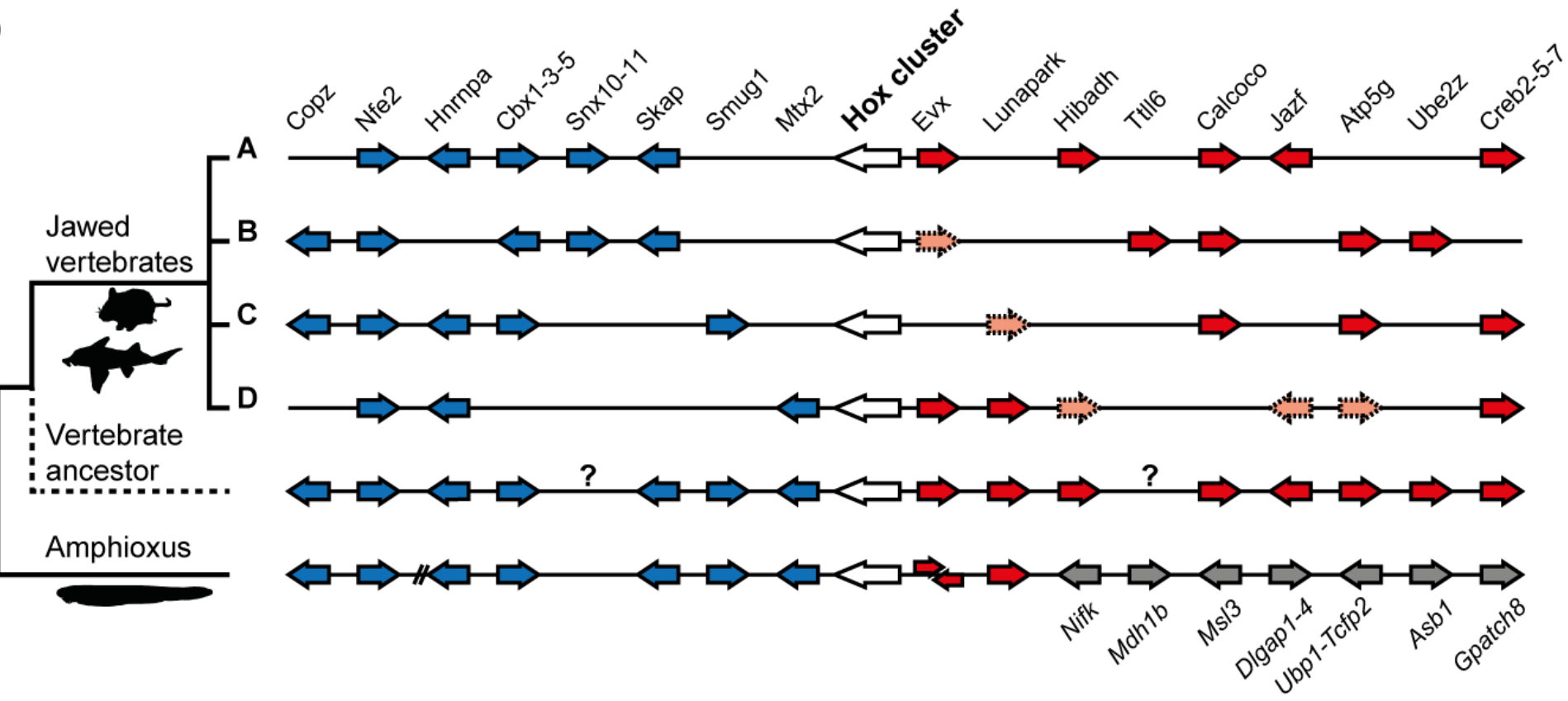



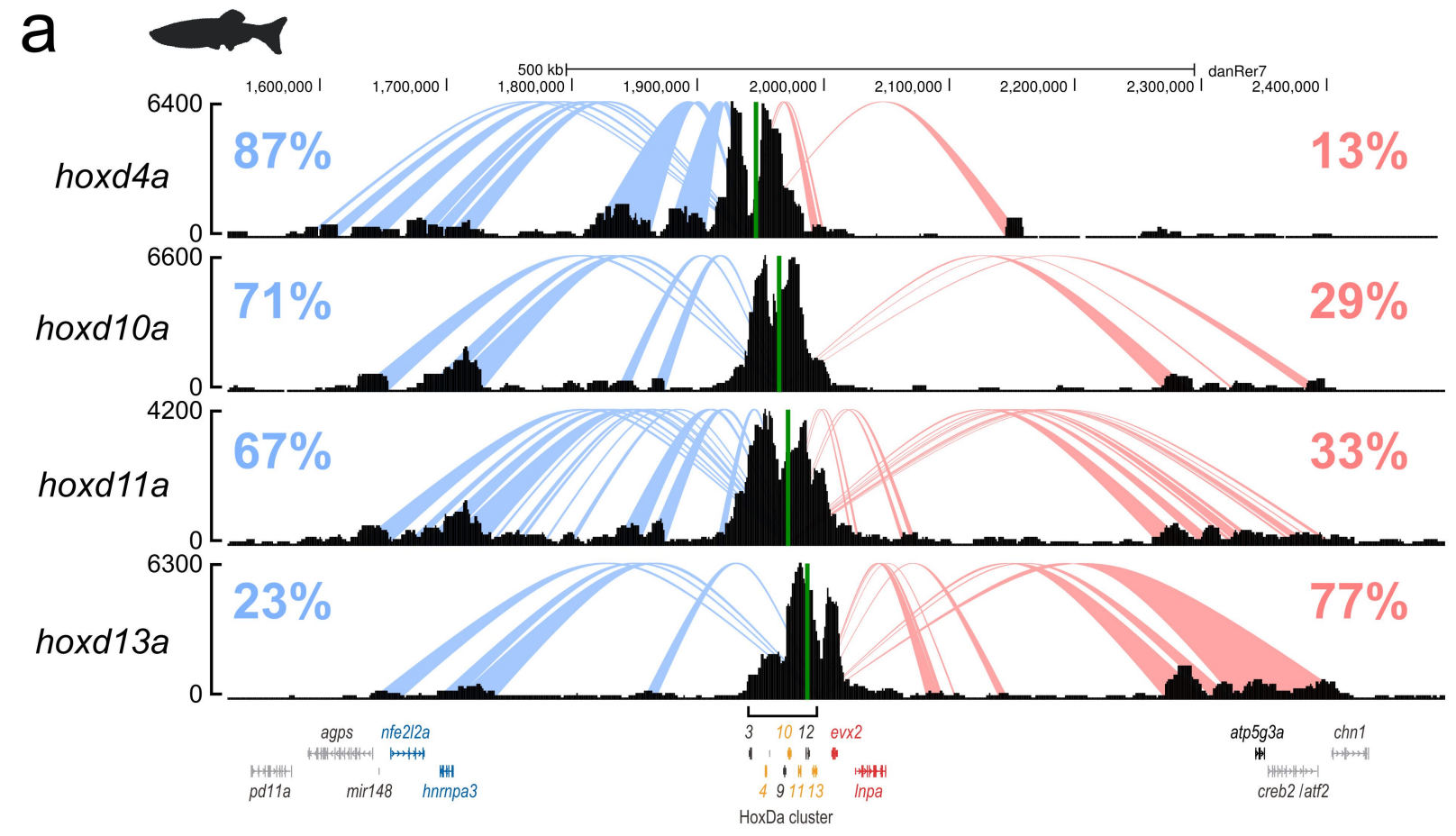

b

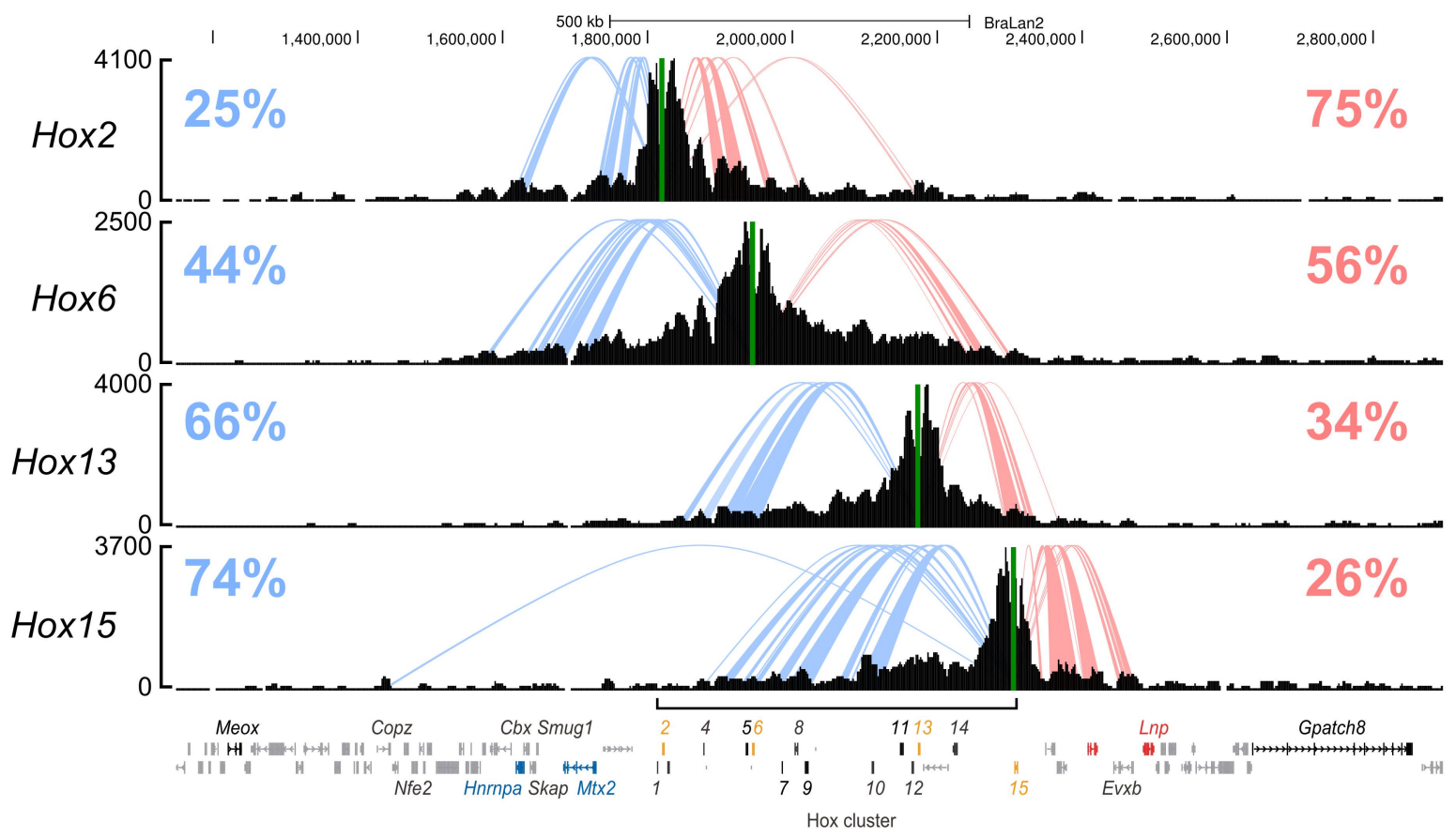


a
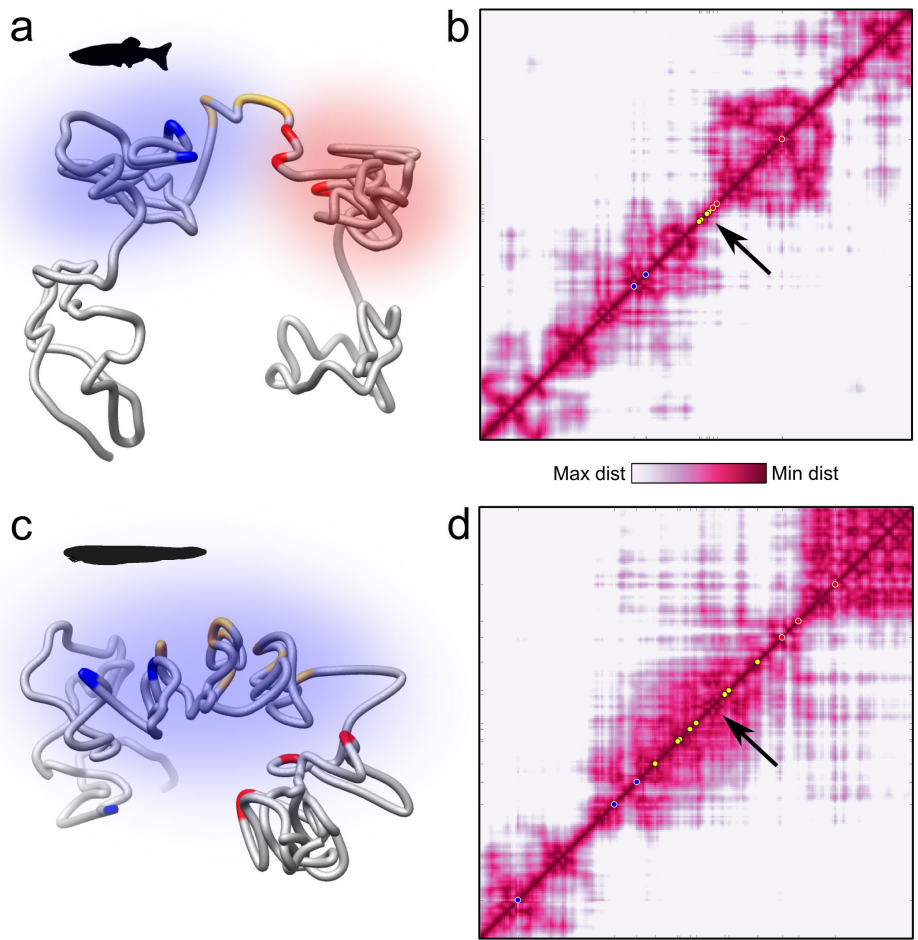


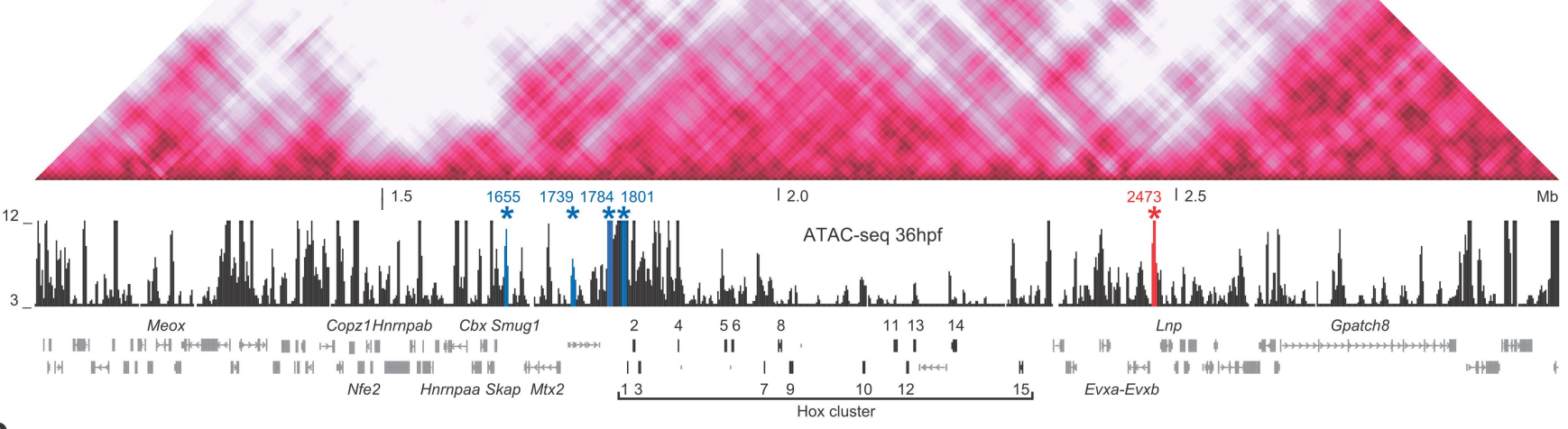

b
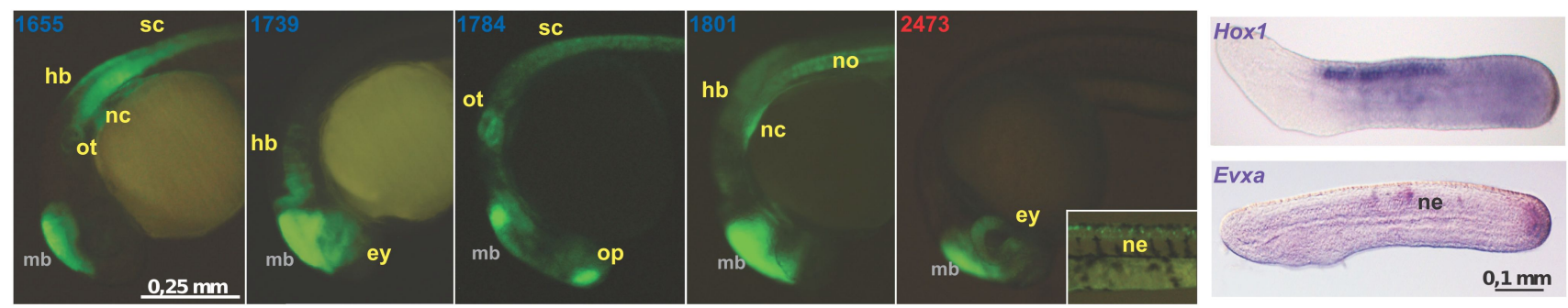

c

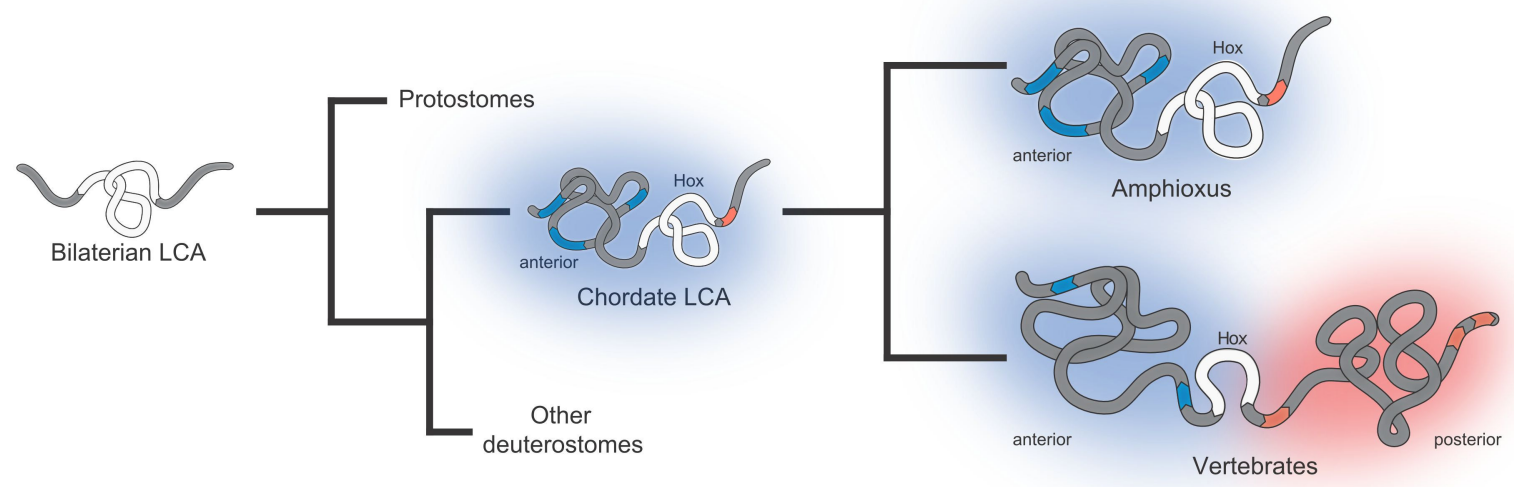

